\title{
A LOWER BOUND FOR THE EXPONENT OF CONVERGENCE OF NORMAL SUBGROUPS OF KLEINIAN GROUPS
}

\author{
JOHANNES JAERISCH
}

\begin{abstract}
We give a short new proof that for each non-elementary Kleinian group $\Gamma$, the exponent of convergence of an arbitrary non-trivial normal subgroup is bounded below by half of the exponent of convergence of $\Gamma$, and that strict inequality holds if $\Gamma$ is of divergence type.
\end{abstract}

\section{IntRoduction AND Statement of RESUlts}

An $(n+1)$-dimensional hyperbolic manifold, for $n \in \mathbb{N}$, can be described by the Poincaré disc model $\mathbb{D}:=$ $\left\{z \in \mathbb{R}^{n+1}:\|z\|<1\right\}$ of hyperbolic $(n+1)$-space quotiented by the action of a Kleinian group $\Gamma$, where we recall that a Kleinian group is a discrete subgroup of the group of all isometries of $\mathbb{D}$ with respect to the hyperbolic metric $d$. For each Kleinian group $\Gamma$, we can canonically associate the Poincaré series $P(\Gamma, s):=\sum_{\gamma \in \Gamma} e^{-s d(0, \gamma(0))}$ for each $s \in \mathbb{R}$. The exponent of convergence $\delta(\Gamma)$ of a Kleinian group $\Gamma$ is given by the abscissa of convergence of the associated Poincaré series, that is $\delta(\Gamma):=\inf \{s \geq 0: P(\Gamma, s)<\infty\}$. Furthermore, $\Gamma$ is said to be of divergence type if the series $P(\Gamma, \delta(\Gamma))$ diverges. The quantity $\delta(\Gamma)$ carries information about the complexity of the action of $\Gamma$ at the boundary at infinity $\mathbb{S}:=\left\{z \in \mathbb{R}^{n+1}:\|z\|=1\right\}$ of hyperbolic space. For instance, by a theorem of Bishop and Jones ([BJ97]), it is known that $\delta(\Gamma)$ is equal to the Hausdorff dimension of the radial limit set of $\Gamma$. For more details on Kleinian groups, limit sets and the associated hyperbolic manifolds we refer to [Bea83, Mas88, Nic89, MT98, Str06].

It is of interest to study how the exponents of convergence $\delta(\widehat{\Gamma})$ and $\delta(\Gamma)$ are related for a non-trivial normal subgroup $\widehat{\Gamma}$ of a Kleinian group $\Gamma$. Each normal subgroup $\widehat{\Gamma}$ corresponds to a hyperbolic manifold $\widehat{M}=\mathbb{D} / \widehat{\Gamma}$ which is a normal covering of $M=\mathbb{D} / \Gamma$. In [Bro85] it is shown that if $\Gamma$ is convex cocompact and $\delta(\Gamma)>n / 2$, then $\delta(\widehat{\Gamma})=\delta(\Gamma)$ if and only if the quotient group $\Gamma / \widehat{\Gamma}$ is amenable. Recently, this result was extended to normal subgroups of essentially free Kleinian groups $\Gamma$ with arbitrary exponent of convergence $\delta(\Gamma)$ in [Sta11]. For a recent account of the interplay between the exponent of convergence, the Hausdorff dimension of the limit set and the convex core entropy of Kleinian groups we refer to [FM11].

In this note we give a short new proof of the following theorem which states that the exponent of convergence $\delta(\widehat{\Gamma})$ of a non-trivial normal subgroup $\widehat{\Gamma}$ of a non-elementary Kleinian group $\Gamma$ is bounded below by $\delta(\Gamma) / 2$. This theorem complements the results on the coincidence of $\delta(\widehat{\Gamma})$ and $\delta(\Gamma)$. Before stating the result, recall that a Kleinian group is called non-elementary if its limit set consists of more than two (and hence uncountably many) elements.

Theorem 1.1. Let $\widehat{\Gamma}$ be a non-trivial normal subgroup of a non-elementary Kleinian group $\Gamma$. We then have $\delta(\widehat{\Gamma}) \geq \delta(\Gamma) / 2$. If $\Gamma$ is of divergence type then we have that $\delta(\widehat{\Gamma})>\delta(\Gamma) / 2$.

The first assertion of Theorem[1.1] was obtained by Falk and Stratmann ([FS04, Theorem 2]) using a result of Matsuzaki ([Mat02, Theorem 6]). The complete statement of Theorem[1.1 was proved by Roblin ([ Rob05]).

2000 Mathematics Subject Classification. Primary 30F40; Secondary 37F30.

Key words and phrases. Kleinian groups, exponent of convergence, normal subgroups, hyperbolic geometry.

The author was supported by the research fellowship JA 2145/1-1 of the German Research Foundation (DFG). 
The novelty in our proof of Theorem 1.1 is to show that there exists a uniformly finite-to-one map from a factor of the group $\Gamma$ to the normal subgroup $\widehat{\Gamma}$ (see Lemma 2.2). Combining this with elementary hyperbolic geometry, the first assertion of Theorem 1.1 follows. For the second assertion, we additionally make use of Proposition 2.3, which is taken from [MY09, Corollary 4.3] and which is based on a uniqueness property of the Patterson-Sullivan measure for Kleinian groups of divergence type.

We remark that in certain special cases we are able to simplify the proof of Theorem 1.1 even further by replacing the uniformly finite-to-one map (see the definition prior to Lemma2.2) by a one-to-one map from $\Gamma$ to $\widehat{\Gamma}$ (see Proposition 2.4). These special cases include the case where $\Gamma$ is a free group of rank greater than one and $\widehat{\Gamma}$ is an arbitrary non-trivial normal subgroup of $\Gamma$, as well as all the non-elementary Kleinian groups $\Gamma$ and non-trivial normal subgroups $\widehat{\Gamma}$ such that $\widehat{\Gamma}$ contains a free subgroup of rank two which is a malnormal subgroup of $\Gamma$. We remark that regarding Fuchsian groups $(n=1)$, all torsion-free Fuchsian groups not corresponding to a closed surface are free groups and are therefore covered by Proposition 2.4 Furthermore, by a result of [Kap99], it is known that any non-elementary subgroup of a torsion-free hyperbolic group $\Gamma$ contains a free group of rank two which is malnormal in $\Gamma$. Thus, the second special case of Proposition 2.4 also covers all convex cocompact Kleinian groups $\Gamma$. Using the concept of relative hyperbolicity it is possible to apply Proposition 2.4 to all non-elementary geometrically finite groups $\Gamma$. In the case of Fuchsian groups, we have that geometrically finite groups coincide with finitely generated groups, whereas in higher dimension we only have that geometrically finite groups are finitely generated. However, in the case $n=2$, we can use that for each finitely generated group $\Gamma$ there exists a geometrically finite group $\Gamma^{\prime}$ which is isomorphic to $\Gamma$. Thus, Proposition 2.4 is applicable to $\Gamma^{\prime}$ and all its non-trivial normal subgroups. Since the result of Proposition 2.4 is in the setting of abstract groups, it also applies to all non-trivial normal subgroups of all non-elementary finitely generated Kleinian groups in dimension $n=2$. It would be interesting to know if Proposition 2.4 can be extended to all non-elementary Kleinian groups (see Problem 2.5).

Recently, the results stated in Theorem 1.1 were partially obtained in [BTMT12] in the special case that $\Gamma$ is convex cocompact. The proof given in there uses ergodicity of the geodesic flow on $\mathbb{D} / \Gamma$ and makes use of a result of Lundh ([Lun03]). In [BTMT12], it is also shown that the lower bound in Theorem[1.1] is sharp in the sense that for certain groups of the first kind $\Gamma$, there exists a sequence of normal subgroups $\left(\widehat{\Gamma}_{n}\right)_{n \in \mathbb{N}}$ of $\Gamma$ such that $\lim _{n \rightarrow \infty} \delta\left(\widehat{\Gamma}_{n}\right)=\delta(\Gamma) / 2$.

The existence of a finite-to-one map from a factor of $\Gamma$ to the normal subgroup $\widehat{\Gamma}$, which is employed in this paper, was also used in the author's doctoral dissertation ([Jae11b, Theorem 6.2.10]) in order to obtain the first assertion of Theorem 1.1 for normal subgroups of Fuchsian groups of Schottky type. Furthermore, this idea was used to obtain the analogue of Theorem 1.1 in the context of fractal models of normal subgroups of Schottky groups in [Jae11a, Theorem 1.2]. Recently, the author has extended Proposition 2.3, which was vital in the proof of the second assertion of Theorem 1.1 in terms of the thermodynamic formalism of group-extended Markov systems ([ذ]

Let us end this introduction with a brief discussion of further generalisations of Theorem 1.1 to isometry groups of CAT $(-1)$ spaces and Gromov hyperbolic groups. Matsuzaki and Yabuki have informed the author about work in progress which extends Proposition 2.3 to isometry groups acting properly discontinuously on a proper CAT $(-1)$ space, and quasiconvex cocompact hyperbolic groups. Provided that an extension of Proposition 2.3 holds, and that Proposition 2.4 applies, our proof of Theorem 1.1 can be verbatim extended to isometry groups of a CAT $(-1)$ space and Gromov hyperbolic groups. As an example, consider the free group $\Gamma$ with at least two generators, which acts isometrically on its Cayley graph $(X, d)$ with respect to 
the word metric $d$. Then $(X, d)$ is a CAT $(-1)$ space and Theorem 1.1 holds for each non-trivial normal subgroup of $\Gamma$. It would be interesting to know to what extent Proposition 2.3 can be further generalised.

Acknowledgement. The author would like to thank Professor Matsuzaki for inviting him to Waseda University and for the warm hospitality during his stay in Tokyo, where this paper was finished. The author is grateful for fruitful discussion with Professor Matsuzaki on the subject of this paper, bringing the relative Poincaré series and the concept of malnormal subgroups to the author's attention. The author thanks an anonymous referee for his valuable suggestions on an earlier version of this paper.

\section{Proof of THE THEOREM}

Let us begin by proving an elementary lemma which allows us to investigate the Poincaré series in terms of a certain relative Poincaré series. For the hyperbolic metric $d$ and $A \subset \mathbb{D}$ we set $d(0, A):=\inf _{x \in A} d(0, x)$. For a subgroup $H$ of a group $G$ we denote by $H \backslash G$ the set of right cosets $\{H g: g \in G\}$ and for each $g \in G$ we set $[g]:=H g$.

Lemma 2.1. Let $\Gamma$ be a non-elementary Kleinian group. For each hyperbolic element $h \in \Gamma$ and for each $s>0$ there exists a constant $C>0$ such that

$$
\sum_{\gamma \in \Gamma} \mathrm{e}^{-s d(0, \gamma(0))} \leq C \sum_{[g] \in\langle h\rangle \backslash \Gamma} \mathrm{e}^{-s d(0,[g](0))} .
$$

Proof. Without loss of generality we may assume that the origin is an element of the axis $C(h)$ of the hyperbolic element $h$ which joins the fixed points $p$ and $q$ of $h$. For each $[g] \in\langle h\rangle \backslash \Gamma$ we choose $g_{0} \in[g]$ such that the orthogonal projection $P_{0}$ of $g_{0}(0)$ on $C(h)$ satisfies $d\left(0, P_{0}\right) \leq d(0, h(0))$. We then clearly have that

$$
d(0,[g](0)) \leq d\left(0, g_{0}(0)\right) \leq d(0, h(0))+d\left(P_{0}, g_{0}(0)\right) .
$$

Using this estimate, it follows for each $n \in \mathbb{Z}$ that the orthogonal projection $P_{n}$ of $h^{n} g_{0}(0)$ on $C(h)$, which is given by $P_{n}=h^{n} P_{0}$, satisfies

$$
d\left(P_{n}, h^{n} g_{0}(0)\right)=d\left(P_{0}, g_{0}(0)\right) \geq d(0,[g](0))-d(0, h(0)) .
$$

By the First Law of Cosines ([Rat06, Theorem 3.5.3] for right-angled hyperbolic triangles we can estimate for each $n \in \mathbb{Z}$ that

$$
d\left(0, h^{n} g_{0}(0)\right)+2 \log 2 \geq d\left(0, P_{n}\right)+d\left(P_{n}, h^{n} g_{0}(0)\right) .
$$

Combining the previous inequality with the fact that $d\left(0, P_{n}\right) \geq(|n|-1) d(0, h(0))$ and using the inequality in (2.1) we conclude for each $n \in \mathbb{Z}$ that

$$
\begin{aligned}
d\left(0, h^{n} g_{0}(0)\right)+2 \log 2 & \geq(|n|-1) d(0, h(0))+d(0,[g](0))-d(0, h(0)) \\
& =(|n|-2) d(0, h(0))+d(0,[g](0))
\end{aligned}
$$

Consequently, we obtain for each $s>0$ and for all $n \in \mathbb{Z}$ that

$$
\mathrm{e}^{-s d\left(0, h^{n} g_{0}(0)\right)} \leq 2^{-2 s} \mathrm{e}^{-(|n|-2) d(0, h(0))} \mathrm{e}^{-s d(0,[g](0))} .
$$

Summing the previous inequality over $n \in \mathbb{Z}$ shows that there exists a constant $C>0$ depending on $s>0$ and $h \in \Gamma$ such that for each $[g] \in\langle h\rangle \backslash \Gamma$ we have

$$
\sum_{\gamma \in[g]} \mathrm{e}^{-s d(0, \gamma(0))}=\sum_{n \in \mathbb{Z}} \mathrm{e}^{-s d\left(0, h^{n} g_{0}(0)\right)} \leq C \mathrm{e}^{-s d(0,[g](0))} .
$$

Finally, summing over $[g] \in\langle h\rangle \backslash \Gamma$ in (2.3) finishes the proof of the lemma. 
For each normal subgroup $\widehat{\Gamma}$ of $\Gamma$ and for each $h \in \widehat{\Gamma}$ we consider the map $\iota_{h}: \Gamma \rightarrow \widehat{\Gamma}$ which is given by $\iota_{h}(g):=g^{-1} h g$, for each $g \in \Gamma$. Since for each $n \in \mathbb{Z}$ and $g \in \Gamma$ we have $\iota_{h}\left(h^{n} g\right)=g^{-1} h^{-n} h h^{n} g=\imath_{h}(g)$, this defines a map $\iota_{h}:\langle h\rangle \backslash \Gamma \rightarrow \widehat{\Gamma}$. The next lemma shows that this map is uniformly finite-to-one.

Lemma 2.2. Let $\widehat{\Gamma}$ be a non-trivial normal subgroup of a non-elementary Kleinian group $\Gamma$. For each hyperbolic element $h \in \widehat{\Gamma}$ there exists a constant $k \in \mathbb{N}$ such that the map $\iota_{h}:\langle h\rangle \backslash \Gamma \rightarrow \widehat{\Gamma}$ is at most $k$-to-one.

Proof. Let $p$ and $q$ denote the fixed points of $h$ and let $H$ denote the subgroup of $\Gamma$ which preserves the fixed points of $h$, that is, $H:=\{g \in \Gamma: g(\{p, q\})=\{p, q\}\}$. Since the limit set of $H$ is equal to $\{p, q\}$, we have that $H$ is an elementary group containing the hyperbolic element $h$. Therefore, $H$ is a finite extension of the cyclic group $\left\langle h_{0}\right\rangle$ generated by some hyperbolic element $h_{0} \in H$, and there exists $l \in \mathbb{Z}$ such that $h=h_{0}^{l}$. We conclude that $\langle h\rangle$ is a subgroup of $H$ with finite index $[H:\langle h\rangle]=k-1$, for some $k \geq 2$.

We now show that $\imath_{h}:\langle h\rangle \backslash \Gamma \rightarrow \widehat{\Gamma}$ is at most $k$-to-one. Let $\left[g_{1}\right], \ldots,\left[g_{k+1}\right] \in\langle h\rangle \backslash \Gamma$ be given such that $\iota_{h}\left(\left[g_{1}\right]\right)=\cdots=\imath_{h}\left(\left[g_{k+1}\right]\right)$. Then for each $j \in\{1, \ldots, k\}$ we have that $g_{j}^{-1} h g_{j}=g_{k+1}^{-1} h g_{k+1}$, which implies that $h g_{j} g_{k+1}^{-1}=g_{j} g_{k+1}^{-1} h$. Therefore, for each $j \in\{1, \ldots, k\}$ we have that $g_{j} g_{k+1}^{-1}$ commutes with $h$, which implies that the fixed points of $h$ are preserved by $g_{j} g_{k+1}^{-1}$. Consequently, for each $j \in\{1, \ldots, k\}$ we deduce that $g_{j} g_{k+1}^{-1} \in H$. Since $[H:\langle h\rangle]=k-1$ it follows by the pigeonhole principle that there exist distinct integers $m, n \in\{1, \ldots, k\}$ such that $\left[g_{m} g_{k+1}^{-1}\right]=\left[g_{n} g_{k+1}^{-1}\right]$ in $\langle h\rangle \backslash H$ and hence, $\left[g_{m}\right]=\left[g_{n}\right]$ in $\langle h\rangle \backslash \Gamma$. The proof is complete.

For the sake of completeness we cite the following result from [MY09, Corollary 4.3].

Proposition 2.3. Let $\widehat{\Gamma}$ denote a Kleinian group of divergence type and let $\Gamma$ be a Kleinian group which contains $\widehat{\Gamma}$ as a normal subgroup. We then have that $\delta(\widehat{\Gamma})=\delta(\Gamma)$.

We are now in the position to prove the main theorem.

Proof of Theorem 1.1. Since $\Gamma$ is non-elementary and $\widehat{\Gamma}$ is a non-trivial normal subgroup of $\Gamma$, it follows that the limit sets of $\Gamma$ and $\widehat{\Gamma}$ coincide. Hence, $\widehat{\Gamma}$ is also non-elementary (see e.g. [MT98, Lemma 2.2]). Fix a hyperbolic element $h \in \widehat{\Gamma}$ and let $\iota_{h}:\langle h\rangle \backslash \Gamma \rightarrow \widehat{\Gamma}$ denote the map defined prior to Lemma2.2, which is at most $k$-to-one by Lemma 2.2 for some $k \in \mathbb{N}$. By Lemma 2.1 we have that, for each $s>0$, there exists a constant $C(s)>0$ such that

$$
\sum_{\gamma \in \Gamma} \mathrm{e}^{-s d(0, \gamma(0))} \leq C(s) \sum_{[g] \in\langle h\rangle \backslash \Gamma} \mathrm{e}^{-s d(0,[g](0))} .
$$

An application of the triangle inequality shows that for each $g \in \Gamma$ we have that

$$
\begin{aligned}
d\left(0, g^{-1} h g(0)\right) & \leq d\left(0, g^{-1}(0)\right)+d\left(g^{-1}(0), g^{-1} h(0)\right)+d\left(g^{-1} h(0), g^{-1} h g(0)\right) \\
& =d\left(0, g^{-1}(0)\right)+d(0, h(0))+d(0, g(0))=2 d(g(0), 0)+d(h(0), 0) .
\end{aligned}
$$

From this we deduce that for each $[g] \in\langle h\rangle \backslash \Gamma$ we have

$$
\mathrm{e}^{-d(0,[g](0))} \leq \mathrm{e}^{d(0, h(0)) / 2} \mathrm{e}^{-d\left(0, l_{h}([g])(0)\right) / 2} .
$$

Combining first (2.4) and (2.6) and then using that the map $\imath_{h}:\langle h\rangle \backslash \Gamma \rightarrow \widehat{\Gamma}$ is at most $k$-to-one, we conclude that for each $s>0$,

$$
\sum_{\gamma \in \Gamma} \mathrm{e}^{-s d(0, \gamma(0))} \leq C(s) \mathrm{e}^{s d(0, h(0)) / 2} \sum_{[g] \in\langle h\rangle \backslash \Gamma} \mathrm{e}^{-s d\left(0, t_{h}([g])(0)\right) / 2} \leq k C(s) \mathrm{e}^{s d(0, h(0)) / 2} \sum_{\rho \in \widehat{\Gamma}} \mathrm{e}^{-s d(0, \rho(0)) / 2} .
$$


We can now prove the first assertion of Theorem 1.1. For each $\varepsilon>0$ we have that $\sum_{\gamma \in \Gamma} \mathrm{e}^{-(\delta(\Gamma)-\varepsilon) d(0, \gamma(0))}=$ $\infty$. Hence, it follows by 2.7 that $\sum_{\rho \in \widehat{\Gamma}} \mathrm{e}^{-(\delta(\Gamma)-\varepsilon) d(0, \rho(0)) / 2}=\infty$. Since this holds for each $\varepsilon>0$, we conclude that $\delta(\widehat{\Gamma}) \geq \delta(\Gamma) / 2$. For the proof of the second assertion let $\Gamma$ be of divergence type and suppose by way of contradiction that $\delta(\widehat{\Gamma})=\delta(\Gamma) / 2$. Then it follows by (2.7) that $\widehat{\Gamma}$ is of divergence type. Since $\widehat{\Gamma}$ is a normal subgroup of $\Gamma$, it follows by Proposition 2.3 that $\delta(\widehat{\Gamma})=\delta(\Gamma)$. But from this we deduce that $\delta(\Gamma)=\delta(\Gamma) / 2$ which implies that $\delta(\Gamma)=0$. This gives the desired contradiction, since it is well-known that for each non-elementary group $\Gamma$ we have that $\delta(\Gamma)>0$ (see e.g. [Bea68]). The proof is complete.

2.1. Special cases. In certain special cases the following proposition can further simplify the proof of Theorem 1.1 The next proposition considers abstract groups and might be of independent interest. Recall that a subgroup $H$ of a group $G$ is called a malnormal subgroup of $G$ if for each $g \in G$ with $g \notin H$ we have that $\left(g H g^{-1}\right) \cap H=\{1\}$.

Proposition 2.4. Let $\widehat{\Gamma}$ be a non-trivial normal subgroup of a group $\Gamma$. If $\Gamma$ is free of rank greater than one, or if $\widehat{\Gamma}$ contains a free subgroup $H=\left\langle h_{1}, h_{2}\right\rangle$ of rank two which is a malnormal subgroup of $\Gamma$, then there exists a finite set $F \subset \widehat{\Gamma}$ and a map $\tau: \Gamma \rightarrow F$ such that the map $\imath: \Gamma \rightarrow \widehat{\Gamma}$, given by $\imath(g):=g \tau(g) g^{-1}$, is one-to-one.

Before we turn to the proof of the proposition let us explain how it can be applied in order to simplify the proof of Theorem 1.1 Combining the proposition with the inequality (2.5) in the proof of Theorem 1.1 we can immediately deduce that for each $s>0$,

$$
\sum_{g \in \Gamma} \mathrm{e}^{-s d(0, g(0))} \leq \max _{h \in F} \mathrm{e}^{s d(0, h(0)) / 2} \sum_{\rho \in \widehat{\Gamma}} \mathrm{e}^{-s d(0, \rho(0)) / 2},
$$

which is analogous to the inequality in (2.7) above. We can then follow the proof of Theorem 1.1 after (2.7) and obtain a proof without having to apply Lemma 2.1 and Lemma 2.2

Proof of Proposition 2.4. First, we consider the case that the group $\Gamma=\left\langle\gamma_{i}: i \in I\right\rangle$ with card $\left.(I)\right\rangle 1$ is free. Fix some arbitrary $h_{0} \in \widehat{\Gamma} \backslash\{1\}$ and set $F:=\left\{\gamma_{1}^{ \pm 1} h_{0} \gamma_{1}^{\mp 1}, \gamma_{2}^{ \pm 1} h_{0} \gamma_{2}^{\mp 1}\right\}$. Let $h_{0}$ be given by the reduced word $\gamma_{\omega_{1}}^{\varepsilon_{1}} \cdots \gamma_{\omega_{l}}^{\varepsilon_{l}}$, where $l \in \mathbb{N}, \omega \in I^{l}$ and $\varepsilon \in\{ \pm 1\}^{l}$. We define $\tau: \Gamma \rightarrow F$ as follows. For $g \in \Gamma$, given by the reduced word $\gamma_{\eta_{1}}^{\rho_{1}} \cdots \gamma_{\eta_{m}}^{\rho_{m}}$, where $m \in \mathbb{N}, \eta \in I^{m}$ and $\rho \in\{ \pm 1\}^{m}$, we choose $\alpha \in\left\{\gamma_{1}^{ \pm 1}, \gamma_{2}^{ \pm 1}\right\} \backslash$ $\left\{\gamma_{\eta_{m}}^{-\rho_{m}}, \gamma_{\omega_{1}}^{-\varepsilon_{1}}, \gamma_{\omega_{l}}^{\varepsilon_{l}}\right\}$ and we set $\tau(g):=\alpha h_{0} \alpha^{-1} \in F$. Then, the element $\imath(g)=g \alpha h_{0} \alpha^{-1} g^{-1} \in \widehat{\Gamma}$ is given by the reduced word $\gamma_{\eta_{1}}^{\rho_{1}} \ldots \gamma_{\eta_{m}}^{\rho_{m}} \alpha \gamma_{\omega_{1}}^{\varepsilon_{1}} \ldots \gamma_{\omega_{l}}^{\varepsilon_{l}} \alpha^{-1} \gamma_{\eta_{m}}^{-\rho_{m}} \cdots \cdots \gamma_{\eta_{1}}^{-\rho_{1}}$, which proves that $t$ is one-to-one.

Secondly, we consider the case that $\widehat{\Gamma}$ contains a free subgroup $H=\left\langle h_{1}, h_{2}\right\rangle$ of rank two which is a malnormal subgroup of $\Gamma$. Consider the left coset decomposition of $\Gamma$ with respect to $H$, that is $\Gamma=\bigcup_{j \in J} g_{j} H$, for some index set $J$ and a fixed choice of $\left(g_{j}\right) \in \Gamma^{J}$. Then, for each $g \in \Gamma$, there exist $j \in J, l \in \mathbb{N}$, words $\omega \in\{1,2\}^{l}$ and $\varepsilon \in\{ \pm 1\}^{l}$, such that $g=g_{j} h_{\omega_{1}}^{\varepsilon_{1}} \cdots h_{\omega_{l}}^{\varepsilon_{l}}$ and $h_{\omega_{1}}^{\varepsilon_{1}} \cdots h_{\omega_{l}}^{\varepsilon_{l}}$ is reduced. We then set $F:=\left\{h_{1}, h_{2}\right\}$ and define $\tau: \Gamma \rightarrow F$ as follows. If $\omega_{l}=1$ then we set $\tau(g):=h_{2}$, otherwise we set $\tau(g):=h_{1}$. We now verify that $\imath: \Gamma \rightarrow \widehat{\Gamma}$ is one-to-one. Let $g, g^{\prime} \in \Gamma$ with $\imath(g)=\imath\left(g^{\prime}\right)$ be given. There exist indices $i, j \in J$, integers $l, m \in \mathbb{N}$ and words $\omega \in I^{l}, \eta \in I^{m}, \varepsilon \in\{ \pm 1\}^{l}$ and $\rho \in\{ \pm 1\}^{m}$ such that $g=g_{i} h$ and $g^{\prime}=g_{j} h^{\prime}$ with reduced words $h=h_{\omega_{1}}^{\varepsilon_{1}} \cdots h_{\omega_{l}}^{\varepsilon_{l}}$ and $h^{\prime}=h_{\eta_{1}}^{\rho_{1}} \cdots h_{\eta_{m}}^{\rho_{m}}$. Note that $l(g)=\imath\left(g^{\prime}\right)$ implies that

$$
g_{j}^{-1} g_{i} h \tau(g) h^{-1} g_{i}^{-1} g_{j}=h^{\prime} \tau\left(g^{\prime}\right)\left(h^{\prime}\right)^{-1} \text {. }
$$

Observe that by definition of $\tau$, we have that $h \tau(g) h^{-1}$ and $h^{\prime} \tau\left(g^{\prime}\right)\left(h^{\prime}\right)^{-1}$ are both elements of $H \backslash\{1\}$. Since $H$ is a malnormal subgroup of $\Gamma$, the equality in (2.8) implies that $g_{j}^{-1} g_{i} \in H$ and thus $i=j$. Now (2.8) implies that $h=h^{\prime}$, which gives $g=g_{i} h=g_{i} h^{\prime}=g^{\prime}$. The proof is complete. 
Let us end this note with the following interesting open question.

Problem 2.5. Let $\widehat{\Gamma}$ be a non-trivial normal subgroup of a non-elementary Kleinian group $\Gamma$. Does there exist a finite set $F \subset \widehat{\Gamma}$ and a map $\tau: \Gamma \rightarrow F$ such that the map $\imath: \Gamma \rightarrow \widehat{\Gamma}$, which is for each $g \in \Gamma$ given by $\imath(g):=g \tau(g) g^{-1}$, is one-to-one?

\section{REFERENCES}

[Bea68] A. F. Beardon, The exponent of convergence of Poincaré series, Proc. London Math. Soc. (3) 18 (1968), $461-483$. MR 0227402 (37 \#2986)

[Bea83] A. F. Beardon, The geometry of discrete groups, Graduate Texts in Mathematics, vol. 91, Springer-Verlag, New York, 1983. MR 698777 (85d:22026)

[BJ97] C. J. Bishop and P. W. Jones, Hausdorff dimension and Kleinian groups, Acta Math. 179 (1997), no. 1, 1-39. MR 1484767 (98k:22043)

[Bro85] R. Brooks, The bottom of the spectrum of a Riemannian covering, J. Reine Angew. Math. 357 (1985), $101-114$. MR 783536 (86h:58138)

[BTMT12] P. Bonfert-Taylor, K. Matsuzaki, and E. C. Taylor, Large and small covers of a hyperbolic manifold, J. Geom. Anal. 22 (2012), 455-470.

[FM11] K. Falk and K. Matsuzaki, The critical exponent, the Hausdorff dimension of the limit set and the convex core entropy of a Kleinian group, arXiv:1106.4409v2 (2011).

[FS04] K. Falk and B. O. Stratmann, Remarks on Hausdorff dimensions for transient limit sets of Kleinian groups, Tohoku Math. J. (2) 56 (2004), no. 4, 571-582. MR 2097162 (2005g:30053)

[Jae11a] J. Jaerisch, Fractal models for normal subgroups of Schottky groups, arXiv:1106.0026 1 (2011).

[Jae11b] _ Thermodynamic Formalism for Group-Extended Markov Systems with Applications to Fuchsian Groups, Doctoral Dissertation at the University Bremen (2011).

[Jae12] _ Recurrent group extensions of symmetric potentials have full Gurevič pressure, arXiv:1205.4490v3 (2012).

[Kap99] I. Kapovich, A non-quasiconvexity embedding theorem for hyperbolic groups, Math. Proc. Cambridge Philos. Soc. 127 (1999), no. 3, 461-486. MR 1713122 (2000i:20070)

[Lun03] T. Lundh, Geodesics on quotient manifolds and their corresponding limit points, Michigan Math. J. 51 (2003), no. 2, 279-304. MR 1992947 (2004e:30067)

[Mas88] B. Maskit, Kleinian groups, Grundlehren der Mathematischen Wissenschaften [Fundamental Principles of Mathematical Sciences], vol. 287, Springer-Verlag, Berlin, 1988. MR 959135 (90a:30132)

[Mat02] K. Matsuzaki, Conservative action of Kleinian groups with respect to the Patterson-Sullivan measure, Comput. Methods Funct. Theory 2 (2002), no. 2, 469-479. MR 2038133 (2005h:30083)

[MT98] K. Matsuzaki and M. Taniguchi, Hyperbolic manifolds and Kleinian groups, Oxford Mathematical Monographs, The Clarendon Press Oxford University Press, New York, 1998, Oxford Science Publications. MR 1638795 (99g:30055)

[MY09] K. Matsuzaki and Y. Yabuki, The Patterson-Sullivan measure and proper conjugation for Kleinian groups of divergence type, Ergodic Theory Dynam. Systems 29 (2009), no. 2, 657-665. MR 2486788 (2010h:37097)

[Nic89] P. J. Nicholls, The ergodic theory of discrete groups, London Mathematical Society Lecture Note Series, vol. 143, Cambridge University Press, Cambridge, 1989. MR 1041575 (91i:58104)

[Rat06] J. G. Ratcliffe, Foundations of hyperbolic manifolds, second ed., Graduate Texts in Mathematics, vol. 149, Springer, New York, 2006. MR 2249478 (2007d:57029)

[Rob05] T. Roblin, Un théorème de Fatou pour les densités conformes avec applications aux revêtements galoisiens en courbure négative, Israel J. Math. 147 (2005), 333-357. MR 2166367 (2006i:37065)

[Sta11] M. Stadlbauer, An extension of a theorem of Kesten to topological Markov chains, arXiv:1110.2942 v2 (2011).

[Str06] B. O. Stratmann, Fractal geometry on hyperbolic manifolds, Non-Euclidean geometries, Math. Appl. (N. Y.), vol. 581, Springer, New York, 2006, pp. 227-247. MR 2191250 (2006g:37038)

Department of Mathematics, Graduate School of Science Osaka University, 1-1 Machikaneyama Toyonaka, OSAKA, 560-0043 JAPAN

E-mail address: jaerisch@cr.math.sci.osaka-u.ac.jp 